\title{
TAKING SOCIAL NETWORKS TO THE NEXT LEVEL
}

\author{
Alexiei Dingli, Dylan Seychell \\ Mark Scerri, Brendan Cutajar, Kristian Galea, Saviour Agius, Mark Anthony Cachia, Justin \\ Saliba, Jeffrey Cassar, Erica Tanti, \\ Sarah Cassar, Shirley Cini, Mariya Koleva \\ Faculty of Information and Communication Technology (ICT) \\ University of Malta \\ Msida, MSD 2080, MALTA
}

\begin{abstract}
Since the boom of social networking lead to people using multiple account on many platforms in order to keep in touch with hundreds of contacts, managing one's contacts risks becoming a burden for many users. Following and finding information about friends and family has become an issue too. Guided by these observations and by careful research of existing adaptive web technologies, our team worked on the development of SNAP - an adaptive social network integrator which aimed to amalgamate various social networks (Facebook, Twitter and Flickr) in one adaptive environment, which unobtrusively sorts the users' feed according to his/her preference. To achieve data transfer and authorisation, SNAP uses the newest version of the OAuth protocol. Adaptivity was achieved through statistical filtering. The initial field tests show that the system works, however there is definitely room for improvement in terms of Social Network Integration, and testers generally expressed an interest in the idea of using an adaptive social integrator such as SNAP. On top of this, we will be suggesting a number of improvements which will change the way we use social networks forever.
\end{abstract}

\section{KEYWORDS}

social networking, adaptive systems, adaptive hypermedia systems, social network portal, social network integration

\section{INTRODUCTION}

More and more people use social networking sites (SNS) everyday to interact with their family and friends, and for various reasons a number of these people use more than one social network. People maintain a large number of contacts (friends) on their chosen social networks and as most social networks today offer many functionalities, such as 
status updates, media sharing (text, photo, audio, video), third party applications and games, the users may experience difficulties finding the content in which they are interested.

Another characteristics of SNS usage is that users are not a homogeneous mass but individuals with distinct preferences over which social network is the best for them. These preferences are not static either, and over the time they tend to change in favour of one network or another. Managing several accounts over different portals may become a burden for many users.

For this purpose the SNAP adaptive web portal was developed, allowing the amalgamation of several popular social networks in one integrated environment. SNAP monitors and learns the browsing behaviour of the users and adapts to it, giving prominence to their preferred social networks.

\section{LITERATURE REVIEW}

\subsection{Social Web}

The World Wide Web (WWW) was proposed by (Berners-Lee and Groff, 1992) in their paper named " $W W W$ ". The concept was that of a group of global internet users contributing to a global source but in a very limited manner when compared to the frequency of the content being queried. In this web, users were mostly consumers of information. In their paper 'Teaching Web Development in the Web2.0 Era', Wang and Zahadat claimed that in around 2003 people started to use the web differently and shifted from the "read-only" environment (Wang and Zahadat, 2009).

We were all used to using the web as simple consumers by reading, filling in forms, messaging and using the internet from a fixed location. This shift introduced the concept of participating as well while using the web as end user and thus becoming coproducers(van Wamelen and de Kool, 2008). This gradually enabled us to publish content with more ease and putting the user at the centre of the web (Morato et al, 2008) by making content dependent on the users thus being more dynamic (Hailpern et al, 2009).

The field of Social Web or Social Computing has expanded after the wide scale integration of Web 2.0 and its enabling of users to contribute directly to web content (Margherita et al, 2010). This is a new dimension which brought with it plenty of motivation in the creation of new applications but at the same time opened the doors for various issues. The social web entails online platforms or website which allow individual who have an account or profile to post content (Zhou et al, 2010). This can be in the form of various 
products which were resultant of the web 2.0. The use of these products allow the sharing of information about personal activities and also about the expression of ideas of individuals.

\subsection{Adaptive Systems}

The function of an adaptive system is to offer personalized experience, analyzing the data from the user's interaction with the system (Brusilovsky and Milan, 2007). It aims to improve the organization and presentation of websites (Perkowitz, M. and Etzioni,O., 1997 cited in Mican and Tomai, 2010) by adaptively selecting, prioritizing and manipulating links and content (Brusilovsky and Milan, 2007). The reasons which gave rise to the development of adaptive systems are varied, for instance, to give personalized recommendations (Balabanović, 1997), to personalize learning experience in tutoring systems (Baena, et al., 2000) or even to and even to adapt information for terminally-ill patients (Bental, et al., 2000). Furthermore, there are many approaches to adaptivity. In this review we are only aiming to present overlay user modelling, which is particularly pertinent to our work on SNAP.

The user model is at the heart of an adaptive system. The problem receives prominence in (Brusilovsky and Milan 2007) in a discussion about adaptive educational systems (AES), but the same principles can be used for other adaptive systems as well. (Brusilovsky and Milan 2007) describe an overlay model as a structural model, which presents the domain knowledge as a set, and the user knowledge - as its subset. The elements of the user knowledge are assigned values (boolean, numeric or a probability) to represent the level of knowledge. When the domain knowledge is modelled, what is modelled can broadly be called concepts. The latter can be facts, rules or constraints. Depending on whether these concepts are independent or related, the domain models are vector (set) models or network models respectively.

Evaluating adaptive websites can be a difficult task, which was also pertinent to our research. (Bauer and Scharl 2000) investigate the possibilities for automatic content and structure evaluation of websites and raise important concerns about the validity of manual (subjective) website evaluation, but their techniques were not immediately applicable on the work on SNAP, one of the reasons being that Braun and Scharl's research is aimed at non-adaptive websites.

(Sadat and Ghorbani 2004) propose a hierarchy of features, specifically aimed at the evaluation of adaptive hypermedia systems. The authors classify the features in three main groups: runtime features, technology, and software engineering. Runtime features refer to the way the system works and behaves, technology features include all the algorithms and techniques employed in the system, and software engineering refers to 
features of the software development. These have been useful in the process of evaluating SNAP.

\section{METHODOLOGY}

\subsection{Basic Information and Functionalities}

The front-end layer of the SNAP system, which takes care of the user requests, was designed using layouts. The back-end communicates with the social networks via specialized classes. There is one parent class and all other specialized social network classes extend it. The general functionalities of SNAP are defined in this parent class and the network classes can also present unique functionalities. In its final form, SNAP is designed to support different social networks. For the prototype, we attempted to implement Facebook, Flickr and Twitter. The functionalities available are the following:

- The main program retrieves the user's posts, user's photos and user's inbox from the various social networks. It also has the function to search (e.g. for other users) across multiple search engines.

- The Facebook class retrieves: the user's feed, wall, message inbox, message thread, albums, user's picture. It also allows the user to post on the Wall.

- The Twitter class retrieves the user and home timeline, and the user's received direct messages. It has the functionality to send a direct message to one or more recipients, to search for a user, to update and delete a tweet, to retweet and to delete a tweet.

- The Flickr class has the function to: retrieve photos, display photos in four formats, retrieve information about photos, and search for photos. Activities on Flicker are: commenting, tagging, adding to favourites, adding to gallery, adding to notes. 


\subsection{Front End Specifications}
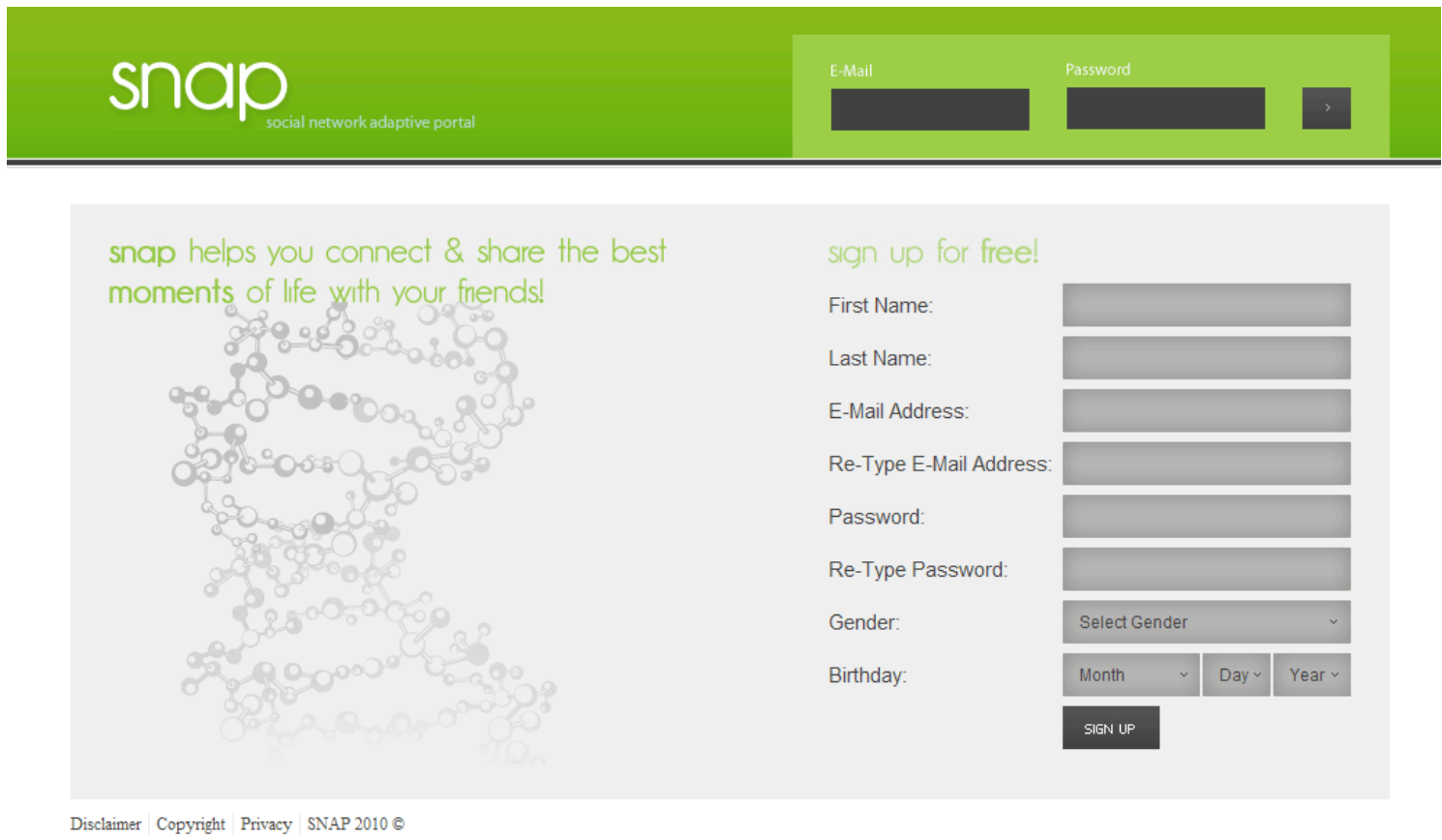

Figure 1: The SNAP login interface

When first coming across the site, a new user registers a new account with the system in a very straight forward procedure as can be seen in Figure 1. As the new user logs in for the first time, they are redirected to a page, where they can manage social networks. Here the user can add the social network accounts they would like to integrate into SNAP (at least one). In the user's homepage, the user's feed is displayed in an ordered fashion. The social network that has the most priority will be displayed first, then the next in priority, and so on until all the spaces on the screen are filled (as can be seen in Figure 2). The user can also choose which particular social network to see using the filter button. At any time the user can delete a social network or add new ones to the system, making SNAP very useful for people who have multiple account on different social networking sites. In the homepage one can find a share section. Here the user can share a status with one or multiple social networks. Messages, photos, and profile edit pages are deprecated in the current version. 


\section{snap}

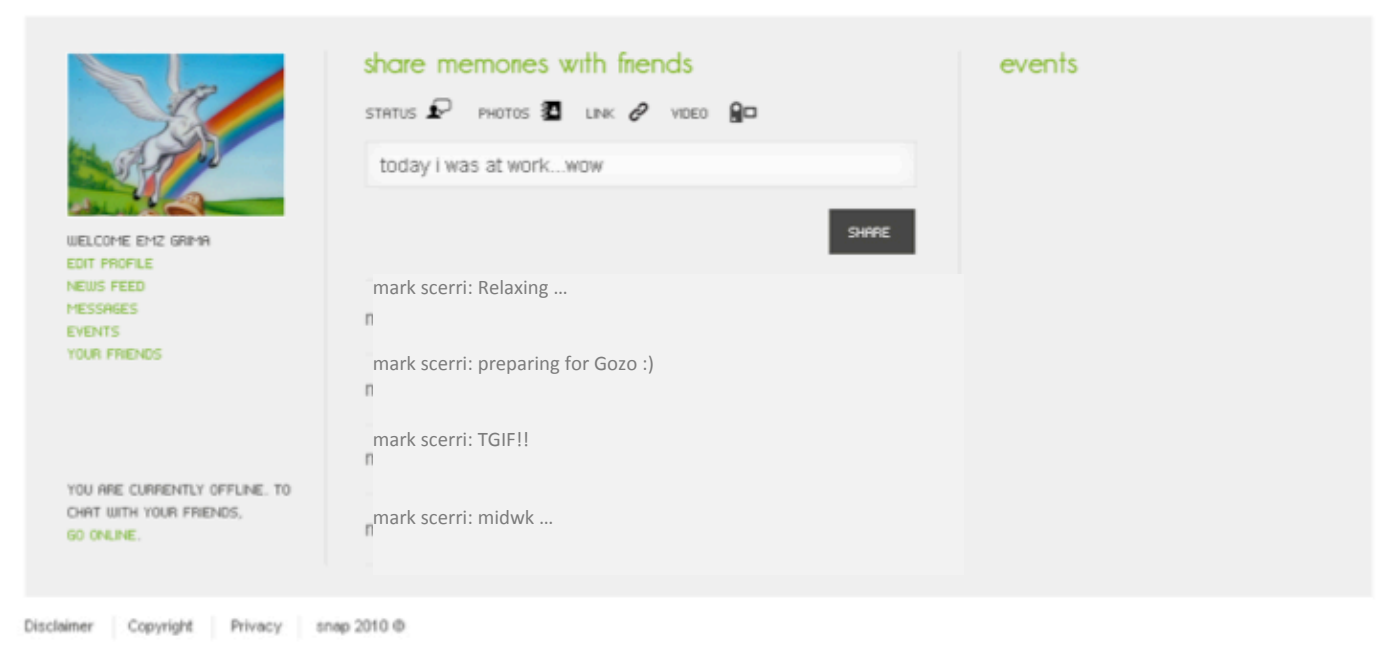

Figure 2: The SNAP main interface

\subsection{Database and Adaptation Implementation}

As in most Web 2.0 applications (O'Reilley 2007), the database is the heart of the system. This project depends entirely on storing the user's social network credentials only once throughout their entire use of the website. Each of the user's account access IDs and tokens are stored within the database for quick access, thus giving the user the need to login only once - and the system handles the rest. The database uses stored procedures to perform functions on itself.

The adaptation is provided by the filter class which is designed to interface the front end with the back end of the system, the front end being all the displayable sections of the solution while the back-end concerns itself with data gathering and representation. It is designed to satisfy the requests from the front end by processing the request made and then executing all the necessary back-end method calls and object creation. Once this is done, the results are gathered and using various algorithms the results are filtered to relay the correct set of elements to the front end for display. This means that this class effectively handles the adaptive section of this solution. 
The filter is specifically intended to use statistical data collected from the use of the site, and use it to make assumptions of the user's preferences. The filters also ensure that it sorts the social networks into the order of preference when displayed on the front end and attempts to allocate more space to the more preferred social network accounts.

The following concerns emerged when it came to displaying features to the front end. There are some social networks which do not receive the same bulk updates that others do. If all the space available is given to the heaviest social network then all other social networks are likely to be given much less prominence than they should. We had to ensure that the user will see most of their preferred material while not omitting other material entirely. To solve this we implemented a filter that retrieves the user's social network accounts from the database and iterates through each, requesting data. The amount of data added to the final result depends entirely on the user's preferences. Thus, a filtering effect is achieved which blocks the less relevant data whilst showing the information which is important to the user.

To produce efficient code whilst reducing maintenance effort, return types as well as method names of each requested feature had to be uniform. The methods that are absolutely necessary are dictated by the parent class which all social networks must extend. Then the return objects are dictated via a set of "generic" classes that ensure that returned objects give the necessary data to the front end of the system.

\subsection{Authorization}

Sharing content and data between SNAP and the social network sites requires authentication, as to ensure confidentiality and integrity of communications. This has been achieved by utilizing the specific APIs of the social networks (when such mechanisms were available) together with the OAuth protocol (Hammer-Lahav, n.d.), which handles the access control. In the prototype, the following APIs were used:

- The Facebook class uses the Graph API - Facebook's social graph view, which contains objects, such as people, photos and events, and the connections between them (e.g., friend relationships, tags, etc) (Facebook, 2011).

- The Twitter class uses the Twitterizer API (Twitterizer, 2011).

- The Flickr class uses the FlickrAPI (Yahoo! Inc., 2011), which consists of methods and API endpoints(Yahoo! Inc., 2011), to send and retrieve data in all of the following formats: REST, XML-RPC, SOAP, JSON, and PHP. 


\section{EVALUATION}

The tests which were planned for SNAP were Black box testing (testing the system with an external view), White box testing (testing whether the correct data constructs are being created during the parsing and receiving of data from social networks), and Field (Alpha) testing, where users were to be asked to fill in a questionnaire to determine their feedback from use of the website. The developers' team tested thoroughly the functionalities of the website using the Black box and White box techniques. In the process, some of the planned features were deprecated in this version of the website.

During the test period, the system displayed correctly in the following browsers: Microsoft Internet Explorer, Mozilla Firefox, Opera and Google Chrome. The system performed without problems on Opera and had some minor issues to display Twitter images in Firefox. Google Chrome displayed the website and the implemented social network functionalities worked fine.

Black box tests were planned on five groups of features of the system: initial log in and registration, integration of a social network account, deletion of a social network account, posting, and photo functionalities. Four out of the planned aspects were tested, with exception of the photo functionalities, since these had to be deprecated from the system.

- The log-in testing involved testing the registration, first-time log in and subsequent log ins. In the final version of the website, the output of the system matched the expected output, with exception of the registration procedure, when during the return to the login page, some formatting errors occurred.

- During the next tests (to add a social network and permissions), Twitter and Facebook accounts were successfully integrated. Flickr, however had some minor issues.

- Tests for deleting a social network account ended up unsuccessful, as the accounts appeared deleted, but in reality persisted. The test was performed on Facebook and Twitter accounts only, for the reasons described in the paragraph above.

- The posting tests meant to establish: whether the system checks for posts in all social networks added, if it checks for posts in a specific networks (chosen by the user), and if sharing a user status across all social networks is visible on all of them. When checking if all posts from a user's social networks are displayed in order of preference, the test was successful (Chrome had some issues with accounting for preference). With regards to specific social networks, the system displayed all posts from the user's social network account in order of most recent with comments, as it was meant to. Sharing a status across all social networks was successful as well. 
White box testing was designed to examine the functions of the system as they were coded. Five functions were examined: Facebook Get Photos, Filter get Posts, Filter get user posts, Facebook getInbox. All the tests were successful.

To complement the tests, the link to the SNAP system was distributed in a Facebook group dedicated to the system which had almost 50 participants. The users were asked to review their initial experience with the system in a questionnaire. They rated the success of social network integration and various site attributes, namely: overall experience, ease of use, social networks functionality, feed, initial account setup, and privacy and security.

The integration Facebook was rated the best and Twitter and Flickr were rated neutral to negative. The site attributes received neutral scores, with exception of the design and layout which received high scores, and the site speed, which received low score. This was due to the fact that the server utilised could not deal with the demands which it was experiencing. The user also left comments, in which they most frequently reported problems with displaying the Twitter timeline. The testers also indicated in their comments that they would be interested to use a social network integrator of this kind. In fact most of them found it very comfortable to view everything the various updates in a single coherent screen. It facilitates and helps them manage their social life.

\section{FUTURE WORK}

Although the present system does provide obvious improvements with regards to managing social networking sites, it is really an aggregator of various feeds. What we're currently working on is to make use of Artificial Intelligence techniques in order to turn it into a powerful knowledge aggregator. The following are the extensions of SNAP which we're currently working on:

- The Semantic Social Graph (SSG). We all know that Social Networking sites have a social graph at the heart of their systems (Iskold 2007). This graph is used to identify the relationships between the various users registered on the site. The SSG is a further layer on top of the social graph aimed at not just showing the relationship between two or more users but it will also give a meaning to those relationships. This will be achieved by using Natural Language Processing techniques such as Sentiment Analysis, Topic Identification, etc. 


\section{Transport}

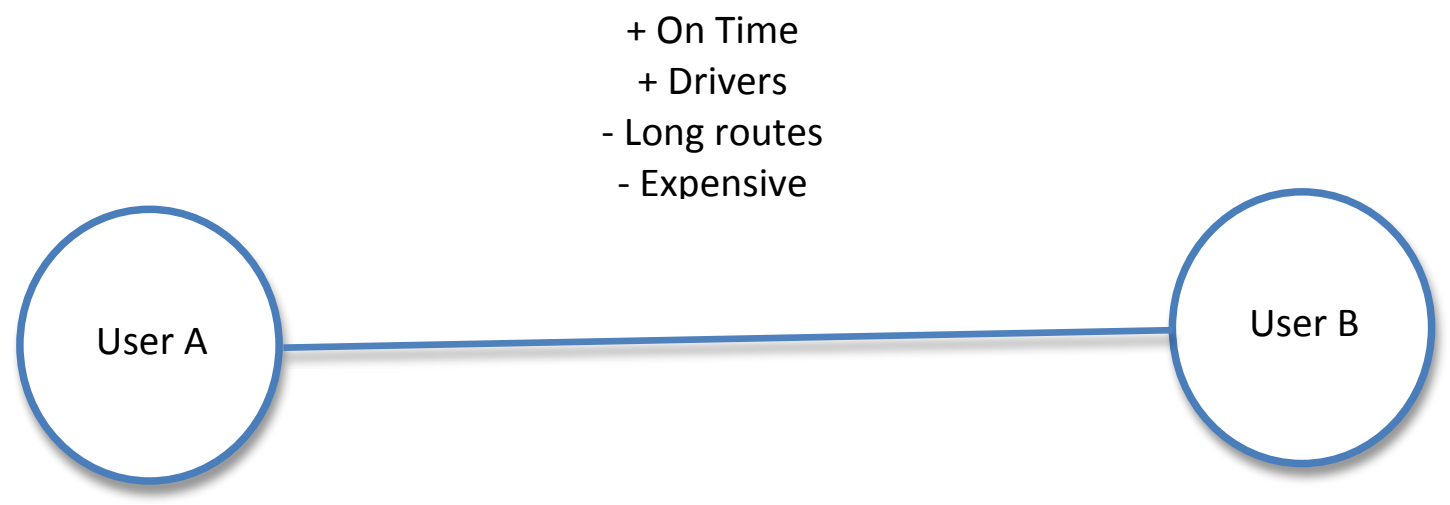

Figure 3: Semantic links between two users

E.g. if two people are discussing via comments the local transport system, by aggregating all the comments exchanged between those two persons, Topic Identification techniques can be used to identify the fact that transportation is the main subject discussed by them at that point in time. Further still, by analyzing the sentiment of those conversations exchanged, we can get a feel of their opinion as can be seen in Figure 3. These are incredibly powerful cues because they can highlight the interest of the person thus helping the system augment the already existing profile whilst also recording the opinion associated to those interests. This information is particularly important when it comes to match people together, since they might be matched according to common interests or opinions thus increasing the value of the social network.

- Expandable Documents. In the world of today where people are faced with the problem of information overload, it is becoming incredibly hard to keep track of information, normally presented in some sort of document (online, offline, etc). Further still, people tend to supplement documents with additional documents thus fuelling further the problem of digesting all the information presented. The Inflatable Document is a structure, which considers a document as being a set of phrases or paragraphs linked together in an ordered way. By making use of Summarization techniques, we will identify the most important sentences in a document and simply present those to the user in a sort of executive summary of the document. If the user requires further information about those documents, he can easily expand them by pressing on them and the information (which is already in the document) but which was previously concealed from him will become available. Further still, when reading particular sections of the document, he can not only expand further inside the current document but by pressing on another button, a right panel will expand whereby snippets from the supporting documents but related to the current snippet in the main document will be 
revealed. These snippets follow the same concept of the main document whereby a click on them will reveal further information. Apart from this, we can also go a step further. The user can request even more details which might not be available to the user. By clicking further on the second set of snippets, another panel will expand to the right which would show snippets from documents harvested on the web. These snippets will be obtained by sending related terms to the most popular search engines, retrieving the top documents and summarizing their content into a sort of executive summary. By using Expandable documents, we will be facilitating the sharing and reuse of information since we will ensure that the users get access to the information they need, when they need it. We will be saving them time and effort since the system will only present them the summary and let them expand on the sections they want at their own pace and thus providing a level of granularity never achieved before. Further still, by making use of Sentiment Analysis we can pinpoint and highlight differences and commonalities in opinions expressed in the document thus helping the users digest the information concealed in the document at a glance.

- Social Mind Maps. Similar maps have been used for centuries because they provide a pictorial way of organizing thoughts thus making it easier for anyone to come up with ideas. Further still, since they are written in a form of document, they can be easily shared with others. The fact that they are online and as part of a social tool will make it even easier to fuel collaborations. This tool will build further on the Semantic Social Graph and on the Expandable documents. When an idea is presented in the form of a Mind Map, the system will look for related documents online and suggest ideas extracted from those documents. This will help augment the mind map with rich information extracted from the web. Further still, the system will act as a sort of broker. By using the sentiment extracted in the Semantic Social Graph, it will also associate people with particular snippets of text thus suggesting them as possible collaborators. This will ensure that the ideas presented in the Mind Map are not simply secluded to the document but they can also be expanded to real people in order to start realizing them.

\section{CONCLUSION}

The SNAP adaptive web portal was developed out of the need to have a system which relieves the user of the burden to visit different social network sites separately in order to maintain contact with their friends, family and colleagues. While amalgamating portals exist, SNAP is designed to also respond to the changing interests and information needs of the users. In other words, SNAP was designed as an adaptive portal which displays the user's social networks in an order of preference that the system learns unobtrusively on its own, with the help of statistical filtering. 
During the final testing by the developers it was discovered that while the core functionalities are in order, there is still room for improvement in terms of social networks functionalities which had to be deprecated, as well as some browser discrepancies. Notwhitstanding this, the users'testing was very positive, something which encourages us to work further on the system.

Future work on the project is currently opening some new avenues for us. We strongly believe that the next evolution of Social Networking sites is to augment them with intelligent techniques borrowed from Artificial Intelligence. By doing so, we would be bringing different people toghether and thus turning Social Networking sites into powerful collaboration tool which make it easier for users to work together whilst being productive.

\section{REFERENCES}

Baena, A. et al., 2000. An Intelligent Tutor for a Web-Based Chess Course. AH '00 Proceedings of the International Conference on Adaptive Hypermedia and Adaptive Web-Based Systems. Springer-Verlag London, UK, pp. 17-26

Balabanović, M., 1997. An Adaptive Web Page Recommendation Service. Proceedings of the First International Conference on Autonomous Agents. Marina del Rey, California, United States, pp.378-385.

Bauer, C and Scharl, A., 2000. Quantitive Evaluation of Web Site Content and Structure'. Internet Research: Electronic Networking Applications and Policy pp. 31-44.

Bental, D. et. al., 2000. AH '00 Proceedings of the International Conference on Adaptive Hypermedia and Adaptive Web-Based Systems. Springer-Verlag London, UK,pp. 27-37

Berners-Lee, T., Cailliau, R., and Groff, J.-F. (1992). The world-wide web. Computer Networks and ISDN Systems, 25(4-5):454-459.

Brusilovsky, P. and Milan, E., 2007. User Models for Adaptive Hypermedia and Adaptive Educational Systems. In: Brusilovsky, P. et. al.,eds. The Adaptive Web. SpringerVerlag, Berlin Heidelberg, pp. 3-53.

Facebook, 2011. Graph API Reference Documentation. Available: http://developers.facebook.com/docs/reference/api/. 
Hailpern, J., Guarino-Reid, L., Boardman, R., and Annam, S. (2009). Web 2.0: blind to an accessible new world. In Proceedings of the 18th international conference on World wide web, WWW'09, pages 821-830, New York, NY, USA. ACM.

Hammer-Lahav, E. et al., n.d. OAuth. Available: http://oauth. net. Last accessed 24th March 2011.

Iskold, Alex. Social Graph: Concepts and Issues. Available: http://www.readwriteweb.com/archives/social_graph_concepts_and_issues.php

Margherita, A., Taurino, C., and Vecchio, P. (2010). Social computing as next-gen learning paradigm A platform and applications. In Elia, G. and Poce, A., editors, Open Networked "i-Learning", pages 91-109. Springer US.

Mican, D. and Tomai, N., 2010. Association-Rules-Based Recommender System for Personalization in Adaptive Web-Based Applications. Proceedings of the 10th international conference on Current trends in web engineering. Vienna, Austria. Springer-Verlag Berlin Heidelberg, pp. 85-90.

Morato, J., Fraga, A., Andreadakis, Y., and Sanchez-Cuadrado, S. (2008). Semantic web or web 2.0? socialization of the semantic web. In Lytras, M. D., Carroll, J. M., Damiani, E., Tennyson, R. D., Avison, D., Vossen, G., and Ordonez De Pablos, P., editors, The Open Knowlege Society. A Computer Science and Information Systems Manifesto, volume 19 of Communications in Computer and Information Science, pages 406-415. Springer Berlin Heidelberg.

O'Reilley, T. What is Web 2.0: Design Patterns and business models for the next generation of software. International Journal of Digital Economics No. 65, pp. 17-37

Sadat, H. and Ghorbani, A. A., 2004. On the Evaluation of Adaptive Web Systems. Proceedings of the Workshop on Web-Based Support Systems. Beijing, China, pp. 127136.

Twitterizer, 2011. Twitterizer: We Want to Give your App Twitter. Available: http://www.twitterizer.net/.

van Wamelen, J. and de Kool, D. (2008). Web 2.0: a basis for the second society? In Proceedings of the 2nd International Conference on Theory and Practice of Electronic Governance, ICEGOV '08, pages 349-354, New York, NY, USA. ACM. 
Wang, Y. D. and Zahadat, N. (2009). Teaching web development in the web 2.0 era. In Proceedings of the 10th ACM conference on SIG-information technology education, IGITE '09, pages 80-86, New York, NY, USA. ACM.

Yahoo! Inc., 2011. The App Garden. Available:

http://www.flickr.com/services/api/misc.overview.html.

Zhou, Y., Guan, X., Zheng, Q., Sun, Q., and Zhao, J. (2010). Group dynamics in discussing incidental topics over online social networks. Network, IEEE, 24(6):42 -47. 